\title{
Development and Characterization of Isotropic Magnetorheological Elastomer
}

\author{
S. R. Kumbhar ${ }^{1, *}$, Subhasis Maji ${ }^{1}$, Bimlesh Kumar ${ }^{2}$ \\ ${ }^{1}$ School of Engg. \& Technology (SOET), IGNOU, New Delhi, 110068, India \\ ${ }^{2}$ Principal, J.T. Mahajan College of Engg., Faizpur ,425 424, Maharashtra, India \\ *Corresponding Author: kumbharsr@rediffmail.com
}

Copyright $(2013$ Horizon Research Publishing All rights reserved.

\begin{abstract}
Magneto rheological elastomer (MRE) is a new kind of smart materials. Its dynamic mechanical performances can be controlled by controlling an applied magnetic field. MRE is usually used as stiffness - changeable spring in the semi-active vibration absorber. In order to get perfect vibration control effect, low dynamic damping of MRE is needed. This paper presents a new method of fabricating isotropic MREs under normal temperature and pressure conditions. In the absence of a magnetic field, a variety of MR elastomer samples were prepared using Sylard's184 silicone elastomer along with un-annealed electrolytic iron power 500 mesh and 15 micron size. Their dynamic characteristics like fractional change in resonant frequency and fractional change in have been studied. The effects of iron particles and the applied magnetic field were investigated. This study is also expected to provide a good guide for designing and preparing new MR elastomers.
\end{abstract}

Keywords Smart Material, MRE, Curing, Spring Constant, Response Analysis.

\section{Introduction}

Magnetorheological (MR) materials including MR fluids, MR elastomer and MR foams [1] are important branches of smart materials. MRE have good MR effects with, the mechanical properties can be controlled by a magnetic field. They also have good mechanical performance for using rubber as matrix. Recently, MRE attracted considerable interests, and many applications of MRE were reported [2-5]. MRE was used as stiffness-changeable spring in semi-active vibration absorber [6]. Vibration absorber is kind of machine used for vibration control. It has a vibration system inside, including spring and vibration mass. MRE was used as the spring device. When MRE was fixed on the vibration object, the vibration energy will be transferred to the vibration system of the vibration absorber. In order to get perfect vibration control effect, the energy transfer ratio between the energy transferred and the total energy is good.
The low dynamic damping of spring is need to get good energy transfer ratio. So, in order to get perfect vibration control effect, the dynamic damping of MRE should be controlled to lower value.

\section{Experimental}

\subsection{MRE Mould Preparation}

Full-proof process for MRE preparation has not been provided in previous literatures. For preparation of MRE, different types of rubbers were available in market. While selecting a particular type of rubber, its properties and availability were considered. In this work Sylgard's184 silicone elastomer was selected due to its inherent advantages like - it cures at constant rate, regardless of sectional thickness degree of confinement, works in wide temperature range that is from $-45^{\circ} \mathrm{C}$ to $200^{\circ} \mathrm{C}$, post curing is not require at any condition, available easily, shrinkage is minimum at the timing of curing, no exothermal reaction taking place during curing, clean curing process without formation of any byproducts, and having a good dielectic properties.

The Sylgard's184 was available in two parts namely part $\mathrm{A}$, and part B. Part A is base while part B is curing agent. It was recommended to mix $A$ and $B$ in proportion of 10:1.

By taking lots of efforts and observations under expert's guidance, four MRE moulds were fabricated. Two of them were prepared by using $20 \%$ iron powder with $60 \mathrm{~mm} \mathrm{X}$ $20 \mathrm{~mm}$ with $10 \mathrm{~mm}$ thickness and $15 \mathrm{~mm}$ thickness (Block A and Block $\mathrm{A}_{1}$ ). Another two MRE moulds were prepared by using 30\% iron powder with $60 \mathrm{~mm} \mathrm{X} 20 \mathrm{~mm}$ with $10 \mathrm{~mm}$ thickness and $15 \mathrm{~mm}$ thickness Block $\mathrm{B}$ and Block $\mathrm{B}_{1}$ ). Thoroughly mixed Sylgard's184 silicon elastomer has been poured directly into the mould in which it is to be cured.

The moulds were cured in the absence of any magnetic field; hence the prepared MRE moulds have been called as Isotropic MRE. Prepared MRE moulds have been shown in fig.1. 


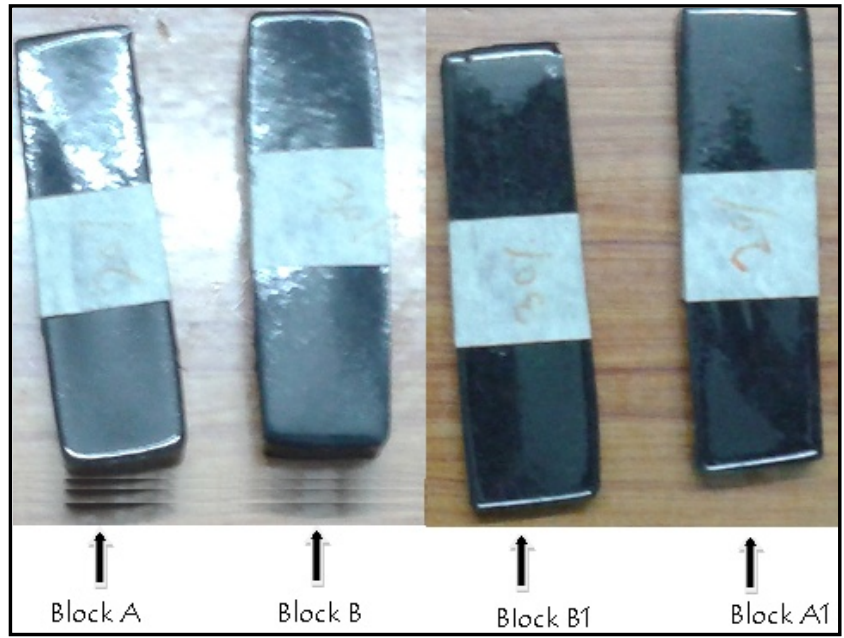

Figure 1. Prepared MRE Moulds

\subsection{MRE Mould Testing}

The test set up (fig.2) for testing fabricated MRE moulds consists of exciter, base plate and accelerometers $\left(A_{1}\right.$ and $\left.A_{2}\right)$. MRE mould was accommodated in aluminum housing with two magnetic poles $\mathrm{M}_{1}$ and $\mathrm{M}_{2}$. Load is applied over top surface of mould. Accelerometer $\mathrm{A}_{2}$ was mounted over base plate which measures the excitations coming from the exciter.

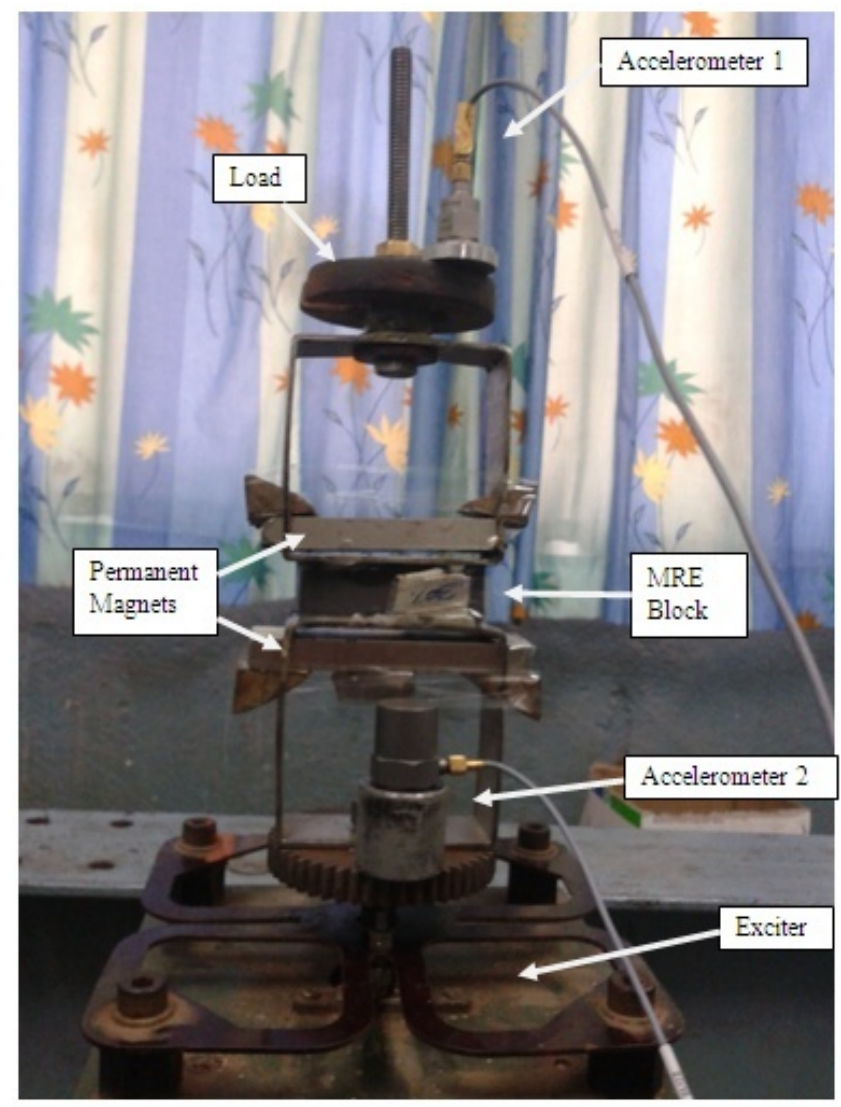

Figure 2. Actual Experimental test set up

While conducting experiments, gain of exciter was adjusted so that exciters output vibration remains constant. Aluminum was used to prepare test set up because it is nonmagnetic material and its presence never affect response of mould in magnetic field. When we removed magnets from the system, we ensured that the overall mass of the system remained the same by adding additional weights to compensate for the magnets. In dynamic response analysis, effect of varying magnetic field on MRE moulds was checked at constant load.

Load was applied over top surface of MRE mould. This load applied were 0,2 and $4 \mathrm{~N}$ respectively for each mould test. Accelerometer was mounted over the load indicating vibration level in absence of magnetic field and in presence of magnetic field for both types of MRE moulds. A magnetic field of $0.12 \mathrm{~T}$ was applied for getting MRE effects.

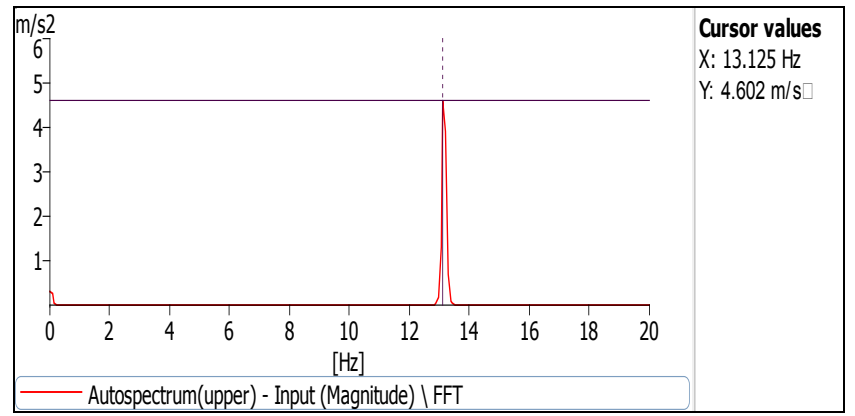

Figure 3. MRE frequency response curve without magnetic field at $0 \mathrm{~N}$ load.

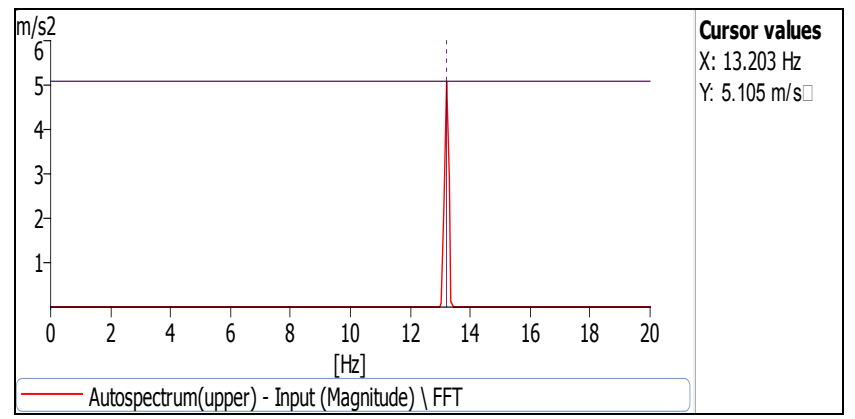

Figure 4. MRE frequency response curve without magnetic field at $2 \mathrm{~N}$ load.

The frequency response of the MRE moulds are shown in figures 3 and 4, with and without application of magnetic field. Each graph contains plot of acceleration versus frequency. X coordinator of cursor value at right side of each figure indicates natural frequency of MRE mould at that particular condition. The natural frequency without magnetic field is indicated by $\mathrm{f}_{0}$ and the same with magnetic field is indicated by $\mathrm{f}_{1}$.

\section{Result, Analysis and Discussions}

During testing frequency responses were taken for different load conditions, for different MRE moulds, with and without applying magnetic field. From the frequency curves, values of frequencies $\mathrm{f}_{0}$ and $\mathrm{f}_{1}$ were recorded.

Percentage changes in resonant frequency were calculated, 
keeping load constant for all the mould conditions, from obtained frequencies.

According to Jolly et.al [5], for an MRE experiencing a shear force, fractional change in modulus $\Delta G / G_{0}$ is related to the fractional change in natural frequency $\Delta \omega / \omega_{0}$; using,

$$
\frac{\Delta \omega}{\omega_{0}}=\sqrt{1+\frac{\Delta G}{G_{0}}}-1
$$

Solving for fractional change in modulus yields,

$$
\frac{\Delta G}{G_{0}}=\left(\frac{\Delta \omega}{\omega_{0}}+1\right)^{2}-1
$$

Assuming that the equation for the shear modulus can be applied to the elastic modulus when the stress is applied uniaxial to the material, the values for the fractional change in modulus have been obtained.

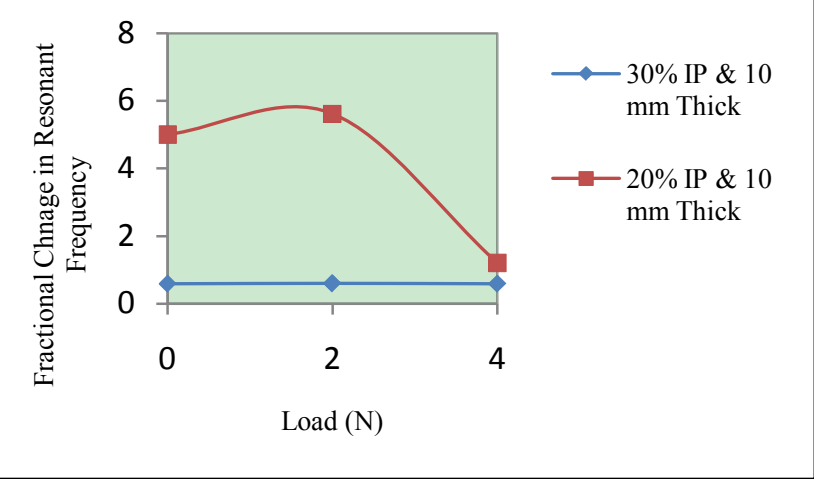

Figure 4. Fractional change in resonance frequency V/s Load $(10 \mathrm{~mm}$ Thick)

The result shown in above graph (fig.4) clearly shows that the fractional change in resonance frequency has been increased by $0.59 \%$ under different loads which indicates improvement in properties of MRE.

The result shown in graph (Fig.5) clearly shows that the fractional change in shear modulus has been increased by $0.187 \%$ to $0.19 \%$ under different loads which indicates improvement in properties of MRE.

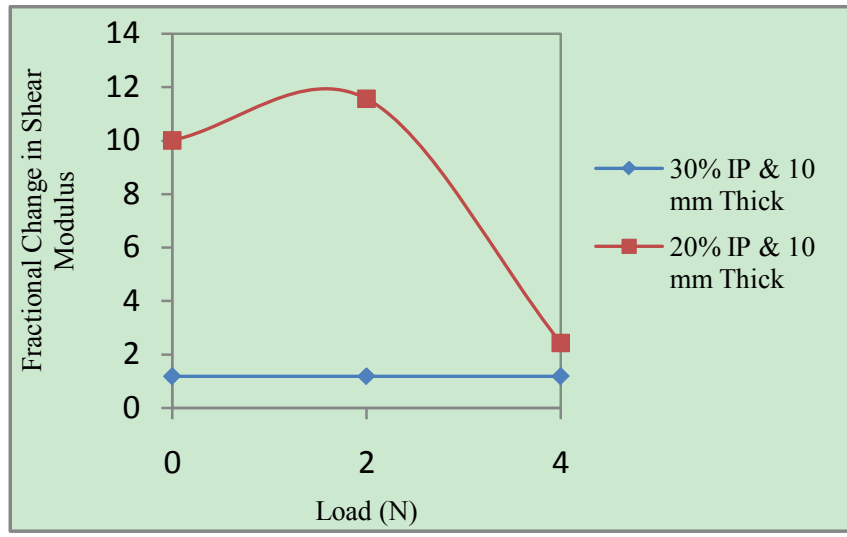

Figure 5. Fractional change in shear modulus Vs load (10 mm Thick)
Similarly the plot of fractional change in resonant frequency and shear modulus for $15 \mathrm{~mm}$ thick MRE moulds were also been plotted (fig. 6 and 7).

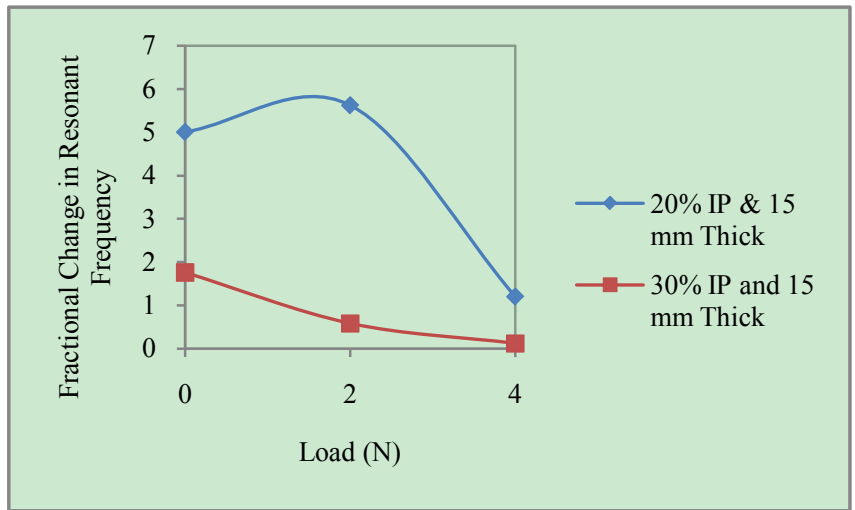

Figure 6. Fractional change in resonance frequency V/s Load $(15 \mathrm{~mm}$ Thick)

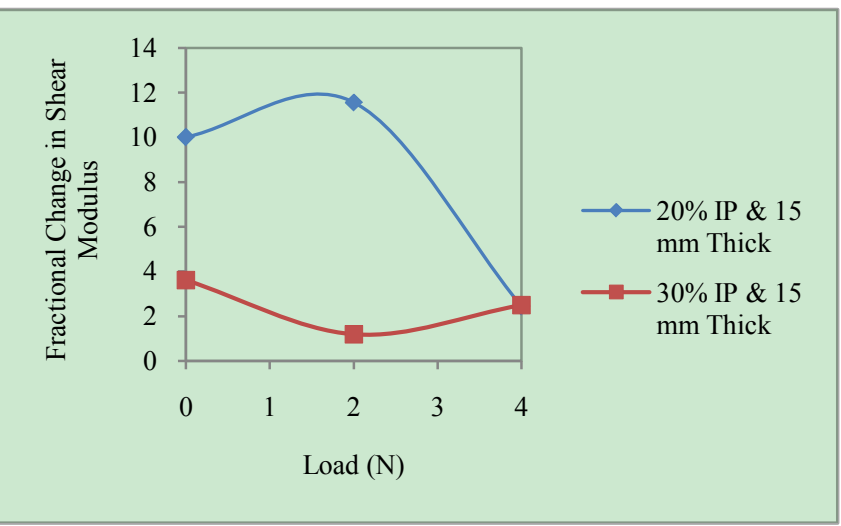

Figure 7. Fractional change in shear modulus Vs load (15 mm Thick)

In case of $15 \mathrm{~mm}$ thick MRE mould, fractional change in resonance frequency is increased by $1.2 \%$ to $5.62 \%$ and fractional change in shear modulus is increased by $2.43 \%$ to $11.56 \%$ with decrease in loads.

By comparing the fractional change in resonant frequency and shear modulus for $20 \%$ iron powder \& $30 \%$ iron powder for $10 \mathrm{~mm}$ thickness MRE mould, the response obtained is shown in fig. 8.

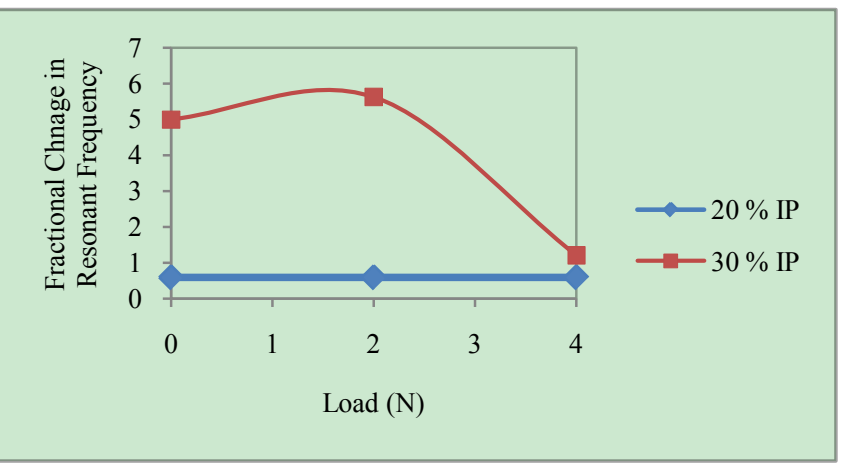

Figure 8. Fractional change in resonant frequency Vs load (20\% IP and $30 \%$ IP $-10 \mathrm{~mm}$ Thick) 


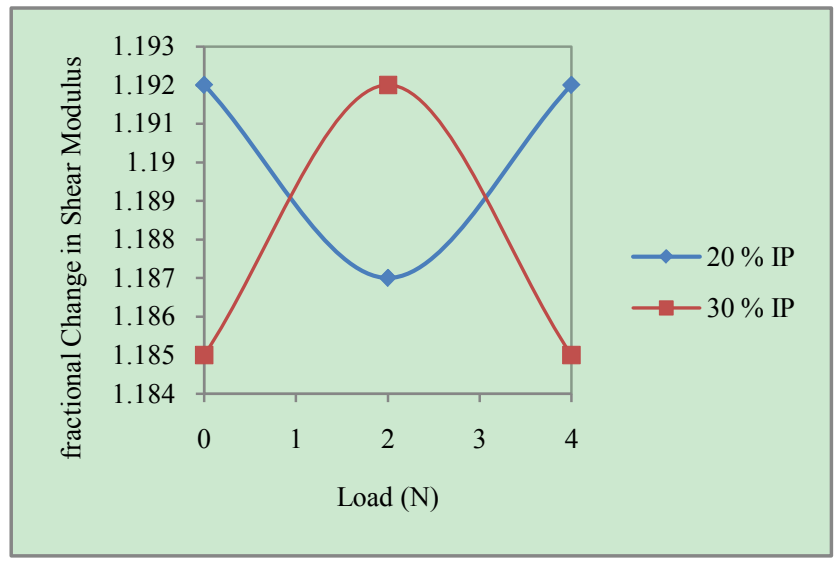

Figure 9. Fractional change in shear modulus Vs load (20\% IP and $30 \%$ IP $-10 \mathrm{~mm}$ Thick)

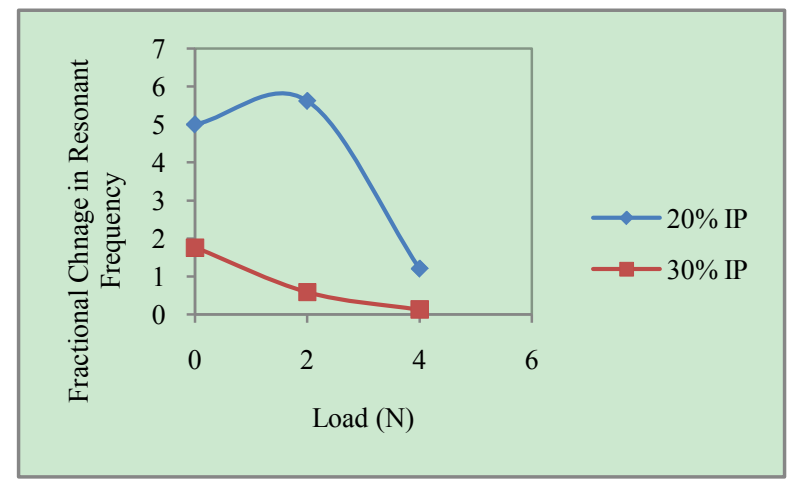

Figure 10. Fractional change in resonant frequency Vs load (20\% IP and $30 \%$ IP -15 mm Thick)

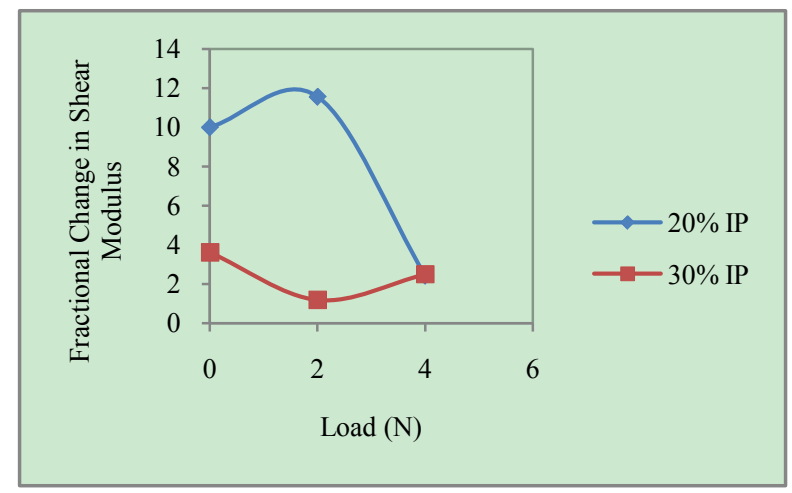

Figure 11. Fractional change in shear modulus Vs load (20\% IP and $30 \%$ IP -15 mm Thick)

From above it is clear that $20 \%$ iron powder MRE mould gives better resonant frequency changes than $30 \%$ configuration. But when we compare shear modulus, it give some conflict between the two. The reason may be, the load applied is not that much high.

Similar comparison has been made between $20 \%$ IP and $30 \%$ IP for $15 \mathrm{~mm}$ thick MRE mould. The graph (fig. 10 and 11) shows the fractional change in resonant frequency and fractional change in shear modulus for $15 \mathrm{~mm}$ thickness MRE moulds.

Also there is significant change of properties due to the content of iron powder. The increase in iron powder does not give necessarily increase in properties. Hence we have to make choice of best percentage of iron powder for getting desired effects.

From above graphs, conclusion can be made that the as we increase the thickness of MRE moulds, there is significant change in corresponding resonant frequency and shear modulus. Both values gets increased, which may be helpful for getting less amount of vibration transmitted to the receiver, if we employ MRE mould in between.

\section{Conclusions}

A new MRE moulds have been developed and tested for its dynamic response under various loads by varying applied magnetic field. The results obtained shows that

- The fractional change in shear modulus and fractional change in resonant frequency increases by increasing applied magnetic field.

- The fractional change in shear modulus and fractional change in resonant frequency increases by increasing applied load.

As the shear modulus and resonant frequency are the functions of stiffness, the prepared MRE moulds will give variable stiffness change, as per the requirement. This property of variable stiffness change can be useful for varying the stiffness of suspension bushing employed in vehicle suspension system, which will lead to fulfill the complicit between ride and handling characteristics of suspension bushing.

In nutshell, MRE could become important feature in automotive sector for reducing noise and vibrations.

\section{Acknowledgments}

Authors are very grateful to Dr. S. G. Joshi, Emeritus Professor, WCE, Sangli for their appropriate and constructive suggestions during preparation of this paper.

\section{REFERENCES}

[1] C. Ruddy, E. Ahearne and G. Byrne, Advanced Manufacturing Science (AMS) Research Centre, Mechanical Engineering, University College Dublin, Belfield, Dublin 4, Ireland (2007).

[2] Hua-xia Deng, Xing-long Gong, Journal of Nonlinear Science and Numerical Simulation, Vol.13 (2008).

[3] H. Bose and R. Roder, $11^{\text {th }}$ International Conference on Electrorheological Fluids and Magnetorheological Suspensions, Dresden, (2008).

[4] Wei Ke-xiang, Meng Guang, Zhang Wen-ming, Zhu Shi-sha, J. Cent. South Univ. Technology, Vol.15 (2008).

[5] M.R.Jolly, J.D.Carlson, B.C.Munoz, T.A.Bullians, Journal of Intelligent Material Systems and Structures, Vol.7 (1996).

[6] Michael Henri, M.S. Thesis, Simon Fraser University, (2005). 